\title{
Unintended consequences of limiting high- frequency emission by small end-user equipment
}

\author{
C.M. Lundmark, E.O.A. Larsson, and M.H.J. Bollen, Fellow, IEEE
}

\begin{abstract}
This paper discusses some consequences of developments in technologies that may impact the risk of electromagnetic interference and equipment damage despite the equipment complying with the tests according to the product standards. Examples of such technologies are: the change from analogue to digital communication, the fast increase in numbers of power converters having smart switching technology and the use of communication via the power system. The paper discusses upcoming sources of emission, uncertainties in emission level, and changing circumstances. It is shown that it is possible for equipment to remain below the emission limits while at the same time the disturbance level increases beyond what was intended by the standard document. The paper also proposes a framework for addressing the problems due to massive penetration of equipment injecting high-frequency harmonics.
\end{abstract}

Index Terms--Electromagnetic compatibility; power quality; conducted disturbances; power-quality and EMC standards.

\section{INTRODUCTION}

$\mathrm{T}$ HE use of power electronics in domestic, commercial and industrial equipment has increased rapidly. There are several advantages with these power converters such as high power density, small losses and low weight. A disadvantage of this type of load is however that it often creates high levels of harmonics. The use of fast switching techniques also creates high-frequency disturbances on the low-voltage grid.

A simple two-pulse diode rectifier forms the interface to the vast majority of computing and consumer-electronics equipment. The resulting current taken from the supply consists of all odd harmonics up to order 9 to 15 [1]. Thirdharmonic currents may be as high as $80 \%$ of the fundamental component. International standards have been introduced to limit the harmonic currents produced by small and large equipment. Other equipment, noticeable energy-saving lamps and some advanced power-electronic driven electrical

\footnotetext{
This work was financially supported by Skellefteå Kraft Elnät AB.

C. M. Lundmark is with the Luleå University of Technology, EMC on Site, 93187 Skellefteå, Sweden (e-mail: martin.lundmark@1tu.se).

E. O. A. Larsson is with the Luleå University of Technology, EMC on Site, 93187 Skellefteå, Sweden (e-mail: anders.1.larsson@ltu.se).

M. H. J. Bollen is the Luleå University of Technology, EMC on Site, 931 87 Skellefteå, Sweden and with STRI AB, 77180 Ludvika, Sweden (e-mail: math.bollen@stri.se).
}

machines, are equipped with a so-called "controlled rectifier" or "active front-end" as interface. These interfaces produce significantly less distortion in the frequency band up to about $1 \mathrm{kHz}$. However they produce instead waveform distortion at higher frequencies, typically at the switching frequency and at harmonics of the switching frequency.

These so-called "high-frequency harmonics" causes new problems. Distribution transformer failure due to high energy spikes and switching transients [2] and disturbances from PWM converters has been reported [3] giving "malfunction of electronic office equipment, blown power supply units, malfunction of electronic controls or unacceptable noise."

This paper will give a brief overview of standards that are currently available to limit emission by power-electronic equipment. The main emphasis of this paper will be, to address the consequences of large-scale penetration of such equipment. Each device itself has been designed to fulfill the standard requirements against minimum costs. The design of equipment has not been aimed at limiting the overall distortion level.

\section{SOURCES OF EMISSION}

\section{A. Harmonics and high-frequency harmonics}

The interest of low weight together with high power density and small losses has resulted in a basic change in power converter construction. Instead of a $50 / 60 \mathrm{~Hz}$ transformer followed of a diode bridge, a storage capacitor and a voltage regulator, the diode bridge is first, followed by a storage capacitor, a dc/ac converter feeding the transformer with a frequency of tens of kilohertz and a voltage step down $\mathrm{dc} / \mathrm{dc}$ converter and regulator.

This solution has besides a number of benefits two basic drawbacks: the fundamental frequency harmonic generation in the input diode rectifier and so called "high-frequency harmonics" from the switching units.

The origin and spread fundamental frequency harmonics and its disadvantages are well known, also some of the solutions like passive and active filters. One of these solutions, power factor correction (PFC), giving opportunity to a wide input voltage range, a displacement power factor close to 1.0 in combination with almost sinusoidal input current.

A passive PFC is often an inductor before the storage 
capacitor smoothing the capacitor charging current. An active PFC uses a switching element together with an inductor, a switched-mode boost converter, before the storage capacitor to force the current, like in a resistor, to follow the shape of the voltage.

The switching frequency and its harmonics in the PFC are generated independently, i.e. uncorrelated, to the frequencies and its harmonics generated in other switching unit's like $\mathrm{dc} / \mathrm{ac}$ converters, dc/dc converters or other PFC, connected to the same power grid. The origin for the PFC switching frequency is also generated independent to the $50 / 60 \mathrm{~Hz}$ fundamental frequency and its harmonic. But, as long as the frequency origin is not the same, two identical converters will differ in switching frequency. These harmonics of the switching frequency are called high-frequency harmonics (HF - harmonics), in distinction from the fundamental frequency harmonics. In the case there is a summation of lots of switching frequencies and their harmonics the expression, high-frequency noise, HF- noise, is also used.

Depending on the construction, the switching frequency in a PFC is rarely absolutely constant but changes continuously depending on variations in input voltage, temperature or loading. In hysteretic control, one of the principles used in SMPS, a hysteretic band around the input voltage is used as a reference. When the instantaneous current reaches the upper or lower limit of the hysteretic band, the current direction is changes by switching. When using hysteretic control, the switching frequency varies during each half period of the ac line voltage. The lowest frequency occurs at the peak of the $\mathrm{AC}$ line and the highest frequency at the zero crossing [4].

In three phase converters used for motor drives it is possible, using PWM techniques, to eliminate harmonics, on the motor side and, in self-commutated converters, also at the line side. The ratio between the PWM modulation frequency and the motor or the grid fundamental frequency, called the frequency ratio, determines the harmonic spectrum for a given pattern of modulation and for a certain degree of control [4]. When the motor speed changes, by changing the motor fundamental frequency, the PWM modulation frequency also changes. "Frequencies generated by self-commutated converters with an operating pulse frequency of several $\mathrm{kHz}$ " have been reported [3] as a source of disturbances in power systems.

The difference in frequency origin, between $50 / 60 \mathrm{~Hz}$ fundamental frequencies and the switching frequency, is an important piece of knowledge in understanding the behavior and the treatments of this switching frequency and its harmonics, to achieve electromagnetic compatibility EMC.

The treatment of the fundamental frequency harmonics is often based on their steady state behavior and the "phase locking" between the three phases and between the harmonics. This makes it possible to create tuned filters at a certain harmonic frequency and to create phase-shift filters using transformer windings.

If the switching, in different sources, takes place at the same frequency but with independent phase angle, tuned filters can still be used to eliminate the switching frequency and at its harmonics. However if the switching frequency varies broadband filters have to be used.

The independent phase angle makes that phase-shift filters can no longer be used. Switching sources generated at the same frequency may add or cancel depending on the phase angle. Different frequencies and independent phase angles results in varying amplitude of the sum signal. This makes characterization, and thus setting of limits, much more complicated.

\section{B. Current disturbances due to multiple devices}

Connecting two or more identical devices using switching techniques to the same supply results in an addition of the conducted disturbances. Fig. 1 shows measurements of the current in the protected earth (PE) wire when one and when two fluorescent tubes using high frequency (HF) ballast are connected.

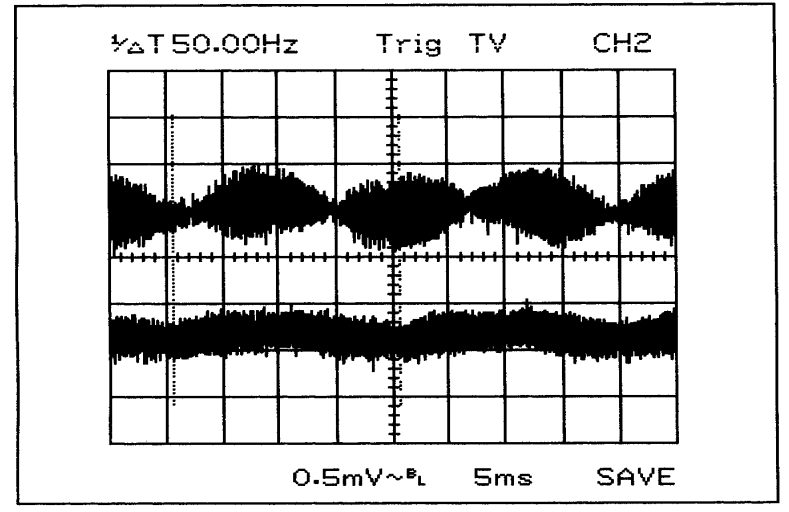

Fig 1. The current in the PE-wire when one, (lower curve) and two (upper curve), HF ballasts are connected to the same supply.

When one HF ballast is connected, lower curve in Fig.1, the high-frequency current is almost stable in amplitude. Note the small $50 \mathrm{~Hz}$ modulation. When two HF- ballasts are connected the small difference in switching frequency gives an amplitude modulation. The amplitude of the highfrequency current varies between zero, when the currents have opposite phase and therefore cancel, and twice the peak to peak value for one ballast, when the two currents are in phase and add.

Mathematically the two switching currents in Fig. 1 could be expressed as an addition of two sinusoidal signals.

Assume the two switching currents $\left(i_{1}, i_{2}\right)$ with the same peak amplitude (I), but with different frequency $\left(f_{l}, f_{2}\right)$

$$
\begin{aligned}
& i_{1}(t)=I \cos \left(2 \pi f_{1} t\right) \\
& i_{2}(t)=I \cos \left(2 \pi f_{2} t\right)
\end{aligned}
$$

where time zero has been chosen such that the two signals have the same initial phase. The sum current equals:

$$
i_{\text {total }}(t)=I\left\{\cos \left(2 \pi f_{1} t\right)+\cos \left(2 \pi f_{2} t\right)\right\}
$$

Using a number of standard trigonometric formulas results in the following expression for the amplitude-modulated signal:

$$
i_{\text {total }}(t)=2 I \cos (2 \pi \Delta f) \cos \left(2 \pi f_{a v}\right)
$$


Where the frequency difference is: $\Delta f=f_{1}-f_{2}$ and the average frequency: $f_{a v}=\frac{1}{2}\left(f_{1}+f_{2}\right)$.

The difference $\Delta f$ between the two switching frequencies appears as a modulation frequency in the upper curve in Fig. 1. The average frequency $f_{a v}$ is not easy to identify in the figure.

When 24 fluorescent tubes using HF ballast are connected to the same supply, as seen in Fig. 2, the modulation becomes stochastic. The worst-case amplitude of the current is, in theory, 24 times the single ballast values. The long-term RMS value is only $\sqrt{24}=4.9$ times the value for a single $\mathrm{HF}$ ballast.

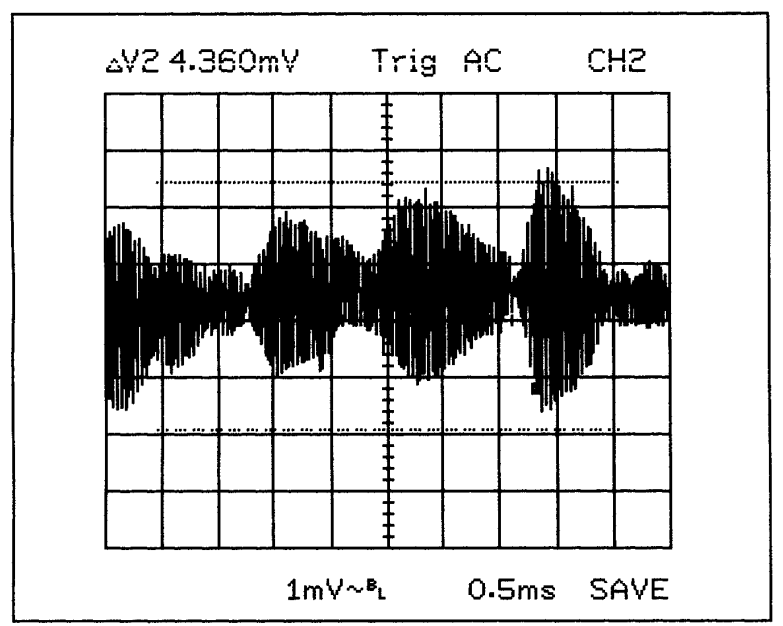

Fig. 2. The current in the PE- wire when 24 HF- ballasts are connected.

The individual switching frequencies in Fig. 1 and Fig. 2 are of almost constant frequency and amplitude with a low level of distortion. If the switching frequencies would be distorted and vary in amplitude and/or frequency the result from 24 switching sources would have been even more complicated to predict.

\section{EXISTING STANDARDS LIMITING HF EMISSION}

Electromagnetic radiation disturbance limits are developed for the purpose of protecting radio communication services. The relevant standards have been developed by taking into account, among others, frequency bands, signal levels, separation distances between the interfering and interfered equipment, desired signal ratio [6].

The radiated emission of electronic equipment is limited with respect to analog radio communication [7], covering the frequency range from the lower limit of the long-wave band $(150 \mathrm{kHz})$ up to the upper limit of the short-wave band (30 $\mathrm{MHz}$ ). The upper limit of $30 \mathrm{MHz}$ also represents the limit for radio communication over the horizon. The quasi-peak detector and the measurement bandwidth $(220 \mathrm{~Hz}$ bandwidth up to $150 \mathrm{kHz}, 9 \mathrm{kHz}$ above $150 \mathrm{kHz}$ ) are chosen with respect to the human hearing, in combination with analog radio communication. The measurement protocol below $150 \mathrm{kHz}$ in CISPR 16-1 is used in military standards covering those frequencies.
Besides radio communication the interest for communication using power lines has increased. Since 1991 very-low-speed power-line communication (PLC) is available in Europe according to a CENELEC standard EN 50065 [8] in a frequency range between 3 to $148.5 \mathrm{kHz}$. Outside of Europe standard IEC 61000-3-8 [9] allows frequencies up to $525 \mathrm{kHz}$. Further interest for power-line communication exists in the frequency range above $1 \mathrm{MHz}$ for broadband over power line (BPL).

Standard document IEC 61800-3 [10] defines the limits for conducted disturbance in the range from $50 \mathrm{~Hz}$ to $2.5 \mathrm{kHz}$ (mains harmonics) and from $150 \mathrm{kHz}$ to $30 \mathrm{MHz}$ (conducted emission) for adjustable-speed drives. European standard EN 55011 [11] defines the main terminal disturbance voltage, measured using a voltage probe or line impedance stabilizing network (LISN) in the range from 9 to $150 \mathrm{kHz}$ for certain types of equipment. Product standards for other devices are under consideration or under preparation. The remaining range from 2.5 to $9 \mathrm{kHz}$ is currently not covered.

The limits and methods of measurement laid down in EN 55011 [11] apply to industrial, scientific and medical (ISM) equipment, and to electro-discharge machining (EDM) and arc welding equipment. Procedures are given for the measurement of radio-frequency disturbances and limits between $9 \mathrm{kHz}$ and $400 \mathrm{GHz}$. Requirements for ISM lighting apparatus operating in the ISM frequency bands of $915 \mathrm{MHz}$ (only allowed in region 2 as defined by the ITU Radio Regulations), $2.45 \mathrm{GHz}$ and $5.8 \mathrm{GHz}$ are contained in this standard. Requirements for other types of lighting apparatus are covered in CISPR 15. [12].

\section{UNCERTAINTIES IN EMISSION LEVELS}

An important aspect of the standards on electromagnetic compatibility is their reproducibility: i.e. two independent testing laboratories should find exactly the same result for the same piece of equipment. The emission levels therefore have to be measured under very precisely defined conditions. A direct consequence of this is that there will always be a discrepancy between the emission level from the test and the actual emission level during the practical application of the device. This deviation is often stochastic in nature and merely requires an additional safety margin between emission and immunity limits (the so-called "compatibility margin"). The "compatibility margin" also handles the summation of emission from several devices.

\section{A. Emission from several devices}

Normally devices are tested, one by one in the laboratory according to the product standard. Tests often concentrate on one disturbance at a time and often only one or a few inputs on the device at a time. To test all inputs and all combinations of disturbance at the same time is rather complicated. It is also complicated to test several units together.

When the tested devices are applied together with other devices, as a source of disturbance, a parallel impedance and as a recipient of disturbance, the disturbance situation 
becomes more complicated. The installation also affects the result; the value of the source impedance is rarely equal to the $50-\Omega$ value used for the standardized test.

Besides the summation of disturbance sources, there is also an apparent risk of impedance mismatch and resonance. This last increases not only the risk for interference, but also for equipment damage.

\section{B. Impedance Mismatch and Resonance}

The insertion loss performance for EMC filters is assuming $50-\Omega$ system impedance ( $50-\Omega$ source impedance, $50-\Omega$ load impedance). The impedance of the power supply is however rarely exactly equal to $50 \Omega$ and further varies with frequency and operating condition of the system. If the impedance of the ac-mains or the impedance of the equipment under test (EUT) differs from $50 \Omega$ the performance of the EMC filter will differ from its expected performance [13].

Another change in filter response occurs due to resonance phenomena associated with impedance mismatch between filter and supply [14] or between filter and EUT [15].

Normally the mains cable between LISN and EUT, during conducted emission (CE) test, has a maximum length of 1 to 2 meters, depending on the particular standard documents. Experiments using an extra $2.5 \mathrm{~m}$ extension cord between LISN and EUT show that this extension cord has a $50 \Omega$ impedance at low frequency, while at higher frequencies the impedance reaches almost $300 \Omega$ [16]. Simulations have shown that a longer distance between the CE at the LISN may impact the conducted emission by more than $10 \mathrm{~dB}$ [17].

\section{CHANGING CIRCUMSTANCES}

In some cases the assumption under which the emission limits were obtained are no longer valid. As mentioned before the underlying aim of the standards is to prevent electromagnetic interference. Setting of an emission limit is just a means for achieving this aim. Due to changing circumstances a situation may arise under which interference occurs despite equipment meeting its emission requirements. In that case, a change in the emission standard is urgently needed. Such a change may involve a reduction of the emission limit, an increase of the immunity limit, or a complete redevelopment of the methods.

\section{A. Introduction of Digital Communication Techniques}

The emission levels for radiated disturbances are based on their potential impact on analog communication systems. When the existing analog technologies are substituted with digital technologies and the number of emitting devices increases, interference could occur even though all equipment complies with the standards.

There is normally no problem when testing the device in the laboratory according to the product standard. But if equipment from different technical areas, i.e. products that have to comply with different product standards, is used in the same power grid, problems can occur especially for higher frequencies. The problems can further increase when different equipment is connected to the same grid and at the same time also connected by communication networks using analogue or digital communications technologies.

There is an increasing interest of communication in the power grid. Dostert [18] shows how spread spectrum techniques has benefit as "special transmission techniques to overcome high and varying levels of interference as well as varying values of impedance and attenuation. "The same spread spectrum techniques are also used in electronic equipment in order to comply with the standard. (See also Section V. C. "Spreading the Disturbance Spectrum")

It is unclear what happens if an increasing part of receiving communication equipment uses spread spectrum techniques to avoid interference at the same time an increasing part of emitting electronic equipment uses spread spectrum techniques to fulfill standard.

\section{B. Switching Frequencies below $150 \mathrm{kHz}$}

The frequency ranges below and above $150 \mathrm{kHz}$ are treated differently in standard documents. The emission requirements above $150 \mathrm{kHz}$ are generally speaking stricter than below 150 $\mathrm{kHz}$. This allows for optimizing the selection of the switching frequency together with the filter sizing: "the switching frequency has been selected so as to have the first and second harmonic component of the ripple below the lower frequency considered by the standards; thus reducing the filter requirements." [19].

The result is that those devices produce a significant amount of conducted disturbances just below the $75 \mathrm{kHz}$ and $150 \mathrm{kHz}$ mark. Further penetration of these devices will not only lead to higher conducted disturbances below $150 \mathrm{kHz}$. It may also cause interference above $150 \mathrm{kHz}$. Consider for example that a large fraction of devices has a switching frequency just below $75 \mathrm{kHz}$, so that the second harmonic falls just outside of the frequency range with stricter limits (i.e. just below $150 \mathrm{kHz}$ ). Their filters will be designed such that the third harmonic of the switching frequency, in this case slightly below $225 \mathrm{kHz}$, falls within the limits. With many such devices installed the result is almost certainly a high level of disturbance in the frequency range $200-225 \mathrm{kHz}$ with potential risk of interference.

\section{Spreading the Disturbance Spectrum}

When electronic equipment is tested in a laboratory according to product standards, the quasi-peak detector [7], is used to measured the conductive and the radiated emission.

The disadvantage in using a fixed switching frequency while using quasi-peak detector in measuring is well known. "...as the period of the signal is fixed, there will always be some energy present at the harmonic frequencies, giving rise in corresponding peaks in the power density spectrum which may lie above the limits set in the EMC standards relevant to the equipment under test." [20].

A common solution is to use a variation of the switching frequency. "Spread spectrum is a well known communications system technique, which may be applied to switched mode 
power converters to reduce the amplitude of the peak unwanted spectral energy present in high order harmonics of a PWM waveform, whilst distributing the energy to additional harmonics" [20].

Random pulse with modulation (RPWM) gives, in motor drives, beside other benefits a "significant reduction of the noise, especially with regard to the quasi-peak values is shown, demonstrating an important advantage of random modulation." [21].

By using spread spectrum, improvements between 6 and 9 $\mathrm{dB}$ measured in quasi-peak have been obtained [20]. If two devices were to be compared, one with fixed and one with spread spectrum, they would produce the same amount of conducted distortion energy. "The total power is the same in the both cases, though the peak level has been reduced" [22]. The power density is lower but not the total power.

Filtering a part of this spread disturbance energy, the total power will be lower, but EMC filtering is only necessarily if the quasi-peak value is too high according to the standard.

However the benefit for the manufacturer of modulated switching frequency is that the device can be equipped with a smaller EMC filter, which will reduce the cost. The result is that both devices produce the same maximum conducted energy per band, but that the total conducted energy for the device with modulated switching frequency may be significantly because of the smaller EMC filter.

The impact on narrowband communication is probably the same, but broadband communication will experience a higher noise level. How this change impacts summation rules and conducted emission is unclear. Note that a varying switching frequency is also obtained by using so-called "hysteretic control" [5].

\section{PROPOSED METHOD TO HANDLED SWITCHING FREQUENCIES BELOW $150 \mathrm{KHz}$}

The last several years the interest for HF harmonics and noise in power systems has increased. One reason is the possibility of communication via the power grid, for internet connection using frequencies between 1and $30 \mathrm{MHz}$ and for communication to energy meters using frequencies between 9 and $95 \mathrm{kHz}$. For both cases increasing levels of HF harmonics could pose a problem.

The power grid is build and optimised for $50 / 60 \mathrm{~Hz}$ fundamental frequencies. The possibility to carry other frequencies differs depending on the grid and on the devices that are connected.

The number of electronic equipment producing HFharmonics has increased at the same time and together with that the number of filters connected to the power grid. These filters are found on the grid-side of every piece of equipment and optimized to fulfill the product standard. The possibility to use the power grid for communication purpose is not considered in those product standards.

The combination of HF-harmonics, often wide spread using different types of modulation, and filters having unknown influence on the power grid transfer impedance, makes an uncertain future for communication using the power grid, particular if with a further increase in the amount of electronic equipment. This problem cannot be solved using better coding techniques only, if communication speed is of interest as well.

If the power grid is to be used as a communication channel this should be taken into considerations in the specification for all equipment connect to the power grid. In other words: it should be considered during the development of product standards.

For example, these specifications could include that only a small number of fixed switching frequencies are allowed and that filter specifications include a low damping for frequencies used for communication versus a high damping for the switching frequencies.

This would not solve the complete problem but it would significantly reduce the complexity of the problem.

\section{CONCLUSIONS}

A fast growing penetration of equipment injecting highfrequency harmonics may lead to incorrect equipment operation or even to damage.

The underlying aim of the EMC standards is to prevent such electromagnetic interference. This underlying aim is implemented by means of a large number of basic EMC standards and product standards. The rarely explicitly written but nevertheless very important assumption is that electromagnetic compatibility is achieved with a high probability when all equipment complies with the product standards.

Most of the emission and immunity tests are made on the device, one by one, in a well-controlled laboratory environment according to the appropriate product standard.

Electromagnetic interference occurs during the application of the device, in a non-controlled environment, often together with hundred or more of other devices, all tested according to their specific product standard. The difference between the controlled laboratory environment and the non-controlled reality makes that interference may occur despite that all equipment and installations comply with the appropriate standards. This is acceptable as long as it is limited to a small number of cases. However changing circumstances make that the probability of interference occurring may increase beyond what is acceptable.

The existing standards for emission of radiated electromagnetic disturbance are developed for the purpose of protecting analog radio communication services using the quasi-peak detector chosen with respect to the human hearing.

The emission requirements above $150 \mathrm{kHz}$ are generally speaking stricter than below $150 \mathrm{kHz}$. This allows for optimizing the selection of switching frequency together with the filter sizing resulting in high levels of "high-frequency harmonics" In the same way, by modulating the switching frequency the disturbance energy is spread over a broad frequency band making it easier to meet the emission 
requirements. The power density is lower but not the total power.

Besides the risk of interference there is a risk for equipment damage. If the amplitudes in HF harmonic current and voltage show a high variation it is possible to have high peak amplitudes, especially with resonances in power grid.

When digital technologies are added to the existing analog technologies and the number of power converters having smart switching technology and other emitting devices increases fast, an interference situation could occur suddenly, especially if the focus of equipment manufacturers is to fulfill the product standard in laboratory.

When the manufacturer optimizes the technical solution according to quality and cost for a device, developing new and smart solutions and still fulfilling the product standard, the interference risk could be higher than intended during the development of the product standard.

It is especially the combinations of all factors, continuously chancing, that could create interference problems. If the technology changes faster than the adjustment of standards the control over electromagnetic interference could be lost.

\section{REFERENCES}

[1] Arrillaga, J. Watson, N.R. Power System Harmonics, second edition. Chichester, England: Wiley, 2003

[2] G Voss, S Mattatia, G Bajog, "Interaction between power electronic components and distribution transformers - Risk evaluation and mitigation techniques" presented at the 18th International Conference on Electricity Distribution Turin, 6-9 June 2005, Paper No. 477.

[3] C Unger, K Krueger, M Sonnenschein and R Zurowski, "Disturbances due to voltage distortion in the $\mathrm{kHz}$ range - Experiences and mitigation measures", presented at the 18th International Conference on Electricity Distribution Turin, 6-9 June 2005, Paper No. 52.

[4] N. Mohan, T. Undeland, W. Robbins, Power Electronics, 2nd ed, Wiley, 1995.

[5] J. Spangler, A. Behera, "A comparison between hysteretic and fixed frequency boost converters used for power factor correction", Applied Power Electronics Conference (APEC'93), San Diego, CA, USA, 7-11 March 1993, pp.281-286.

[6] EN 55011:1998 Industrial, scientific and medical (ISM) radiofrequency equipment-radio disturbance characteristics-Limits and methods of measurements. European Committee for Electrotechnical Standardization (CENELEC), 1998.

[7] CISPR 16-1:1999 Specification for radio disturbance and immunity measuring apparatus and methods, Second edition 1999-10, International Electrotechnical Commission (IEC), 1999.

[8] EN 50065-1:2001 Signaling on low-voltage electrical installations in the frequency range $3 \mathrm{kHz}$ to $148,5 \mathrm{kHz}$ - Part 1: General requirements, frequency bands and electromagnetic disturbances. European Committee for Electrotechnical Standardization (CENELEC), 2001.

[9] IEC 61000-3-8:2002 Electromagnetic Compatibility (EMC) - Part 3: Limits-Section 8: Signalling on low-voltage electrical installationsEmission levels, frequency bands and electromagnetic disturbance levels, International Electrotechnical Commission (IEC), 2002.

[10] IEC 61800-3:1996 Adjustable speed electrical power drive systems Part 3: EMC product standard including specific test methods, International Electrotechnical (IEC) Commission, 1996.

[11] EN 55011:1998 Industrial, scientific and medical (ISM) radiofrequency equipment-radio disturbance characteristics-Limits and methods of measurements. European Committee for Electrotechnical Standardization (CENELEC), 1998.

[12] EN 55011:2004 Industrial, scientific and medical (ISM) radiofrequency equipment-radio disturbance characteristics-Limits and methods of measurements. 2004. Scope of currently ongoing revision as quoted on www.cenelec.org.

[13] Garry, B. Nelson, R. Effect of impedance and frequency variation on insertion loss for a typical power line filter, IEEE International Symposium on Electromagnetic Compatibility, 24-28 Aug. 1998, Volume: 2, p.691 - 695.

[14] Nitta, S. Shimayama, T. Non-resonating noise filter, IEEE International Symposium on Electromagnetic Compatibility, 21-23 Aug. 1990. p.683 - 687 .

[15] Spiazzi, G. Pomilio, J.A. Interaction between EMI filter and power factor preregulators with average current control: analysis and design considerations, IEEE Transactions on Industrial Electronics, Vol. 46, Issue: 3 (June 1999), p.577 - 584

[16] Tsaliovich, A. Moongilan, D. Remote conducted emission testing using matched LISN power cable assembly, IEEE International Symposium on Electromagnetic Compatibility, 21-23 Aug. 1990, p.431- 434

[17] Pignari, S.A.; Orlandi, A.;Long-cable effects on conducted emissions levels, IEEE Transactions on Electromagnetic Compatibility, Vol. 45 Issue: 1 (Feb. 2003), p.43 - 54

[18] Dostert K. M.; Frequency-hopping spread-spectrum modulation for digital communications over electrical power lines, IEEE Journal on Selected Areas in Communications. Vol. 8, no: 4 (May. 1990), p.700 710

[19] Rossetto, L. Buso, S. Spiazzi, G Conducted EMI issues in a 600-W single-phase boost PFC design, IEEE Transactions on Industrial Electronics, Vol.: 36, Issue: 2 (March-April 2000), p.578 - 585.

[20] Stone, D.A. Chambers, B. Howe, D. Easing EMC problems in switched mode power converters by random modulation of the PWM carrier frequency, Applied Power Electronics Conference and Exposition (APEC), 3-7 March 1996, Vol. 1, p.327 - 332

[21] Trzynadlowski, A.M. Zigliotto, M. Bolognani, S. Bech, M.M Reduction of the electromagnetic interference conducted to mains in inverter-fed $\mathrm{AC}$ drives using random pulse width modulation, IEEE Industry Applications Conference, 12-15 Oct. 1998, Vol. 1, p.739 744

[22] Kuisma, M. Variable frequency switching in power supply EMIcontrol: an overview, IEEE Aerospace and Electronic Systems Magazine, Vol. 1, Issue 12 (Dec. 2003), p.18-22.

Martin Lundmark received the MSc degree from the Royal Institute of Technology, Stockholm, Sweden, Currently he is project manager at EMC on Site at Luleå University of Technology, Skellefteå, Sweden. Before joining Luleå University of Technology in 1992 as a Lecturer he was research engineer at $\mathrm{ABB}$ Corporate Research in Västerås

Anders Larsson received the BSc degree from Luleå university of Technology, Skellefteå, Sweden in 1999. Currently he is $\mathrm{PhD}$ student at the same university. Before starting as a PhD student in 2004 he has worked at Luleå University of Technology as research engineer with power quality and EMC issues.

Math Bollen (M'93, SM'98, F'05) received the MSc and $\mathrm{PhD}$ degree from Eindhoven University of Technology, Eindhoven, The Netherlands. Currently he is professor at Luleå University of Technology, Skellefteå, Sweden and manager power quality and EMC at STRI AB, Ludvika, Sweden. Before joining STRI in 2003 he was research associate at Eindhoven University of Technology, Lecturer at University of Manchester Institute of Science and Technology, Manchester, UK and professor in electric power systems at Chalmers University of Technology, Gothenburg, Sweden. 